\title{
A NOTE ON KILLING TORSION OF MANIFOLDS BY SURGERY
}

\author{
STAVROS PAPASTAVRIDIS
}

\begin{abstract}
In this note we prove that every manifold of dimension different than 3, oriented in a bundle theory (for most bundle theories), is cobordant to a manifold which contains not more torsion than the classifying space of the bundle theory.
\end{abstract}

We will give here a generalization (with simpler proof) of a theorem of $\mathbf{R}$. Stong which says that every closed oriented manifold is cobordant, in the oriented sense, to an oriented manifold whose homology contains no odd torsion [3].

Definition 1. If $G$ is an abelian group, then Tor $G$ is the subgroup of $G$ which consists of torsion elements. Let $G=\left(G_{0}, G_{1}, \ldots\right)$ and $H=$ $\left(H_{0}, H_{1}, \ldots\right)$ be graded abelian groups, then we say that "the torsion of $G$ is contained in the torsion of $H$ ", if and only if for every $i$ there is a $j$ such that Tor $G_{i}$ is isomorphic with a subgroup of Tor $H_{j}$.

Let $f_{r}: X_{r} \rightarrow B O(r)$ be a sequence of fibrations with maps $g_{r}: X_{r} \rightarrow X_{r+1}$ such that the usual diagram commutes. For such a situation $R$. Lashof defines the concept of $X$-structure on manifolds (see [2]) and proves a Thomisomorphism for the bordism groups of such manifolds. It is well known that many of the usual classes of manifolds may be described in terms of $X$-structures, e.g. $S O, U$, Spin, etc. We assume that for $r$ big enough $X_{r}$ has a finite number of cells in each dimension, and that the map $\left(g_{r}\right)_{*}: H_{*}\left(X_{r}\right.$; $Z) \rightarrow H_{*}\left(X_{r+1} ; Z\right)$ "stabilizes" (namely for given $n$, it is an isomorphism up to dimension $n$, provided that $r$ is big enough). Finally we assume that $f_{r}^{*}\left(w_{1}\right)=0$, for $r$ very big. Let $H_{*}(X)=$ ind $\lim H_{*}\left(X_{r} ; Z\right)$.

THEOREM 2. Every even dimensional, closed, $X$-manifold is $X$-cobordant to a manifold whose torsion is contained in the torsion of $H_{*}(X)$.

THEOREM 3. If $X_{r}$ is simply connected for $r$ big enough, then every $(4 k+1)$ dimensional $X$-manifold is $X$-cobordant to a manifold whose torsion is contained in the torsion of $H_{*}(X)$.

THEOREM 4. If $X_{r}$ is simply connected for $r$ big enough, and $H_{2 k-1}(X)$ contains no p-torsion for certain prime numbers $p$ (we take $k>1$ ), then every

Received by the editors April 12, 1977 and, in revised form, August 1, 1977.

AMS (MOS) subject classifications (1970). Primary 57D90, 57D65.

Key words and phrases. Cobordism, surgery, torsion. 
$(4 k-1)$-dimensional $X$-manifold is $X$-cobordant to a manifold whose torsion is contained in the torsion of $H_{*}(X)$ in dimensions different than $(2 k-1)$, and in dimension $(2 k-1)$ contains no $p$-torsion for the same prime numbers.

Proof of Theorems 2,3. Let $\nu: M \rightarrow X_{r}$ be the normal map induced by the $X$-structure of $M$. We take $r$ very big. Let $m$ be the dimension of $M$.

By surgery (see Theorem IV.1.13 of [1]) we can make the map $\nu$ an isomorphism in homology up to dimension $[m / 2]-1$, provided $m \geqslant 4$.

If $X_{r}$ is simply connected and $m$ is of the form $(4 k+1)$ (we take $k$ positive), then we can squeeze in an extra dimension to make $\nu$ a monomorphism in homology up to dimension $[\mathrm{m} / 2]$. This is proved exactly like Theorem IV.2.1 of [1].

And the proof follows from Poincare duality and the fact that from the universal coefficient theorem, for any topological space $Y$, we have Tor $H^{i}(Y ; Z)=$ Tor $H_{i-1}(Y ; Z)$. The cases of 1, 2-dimensional manifolds offer no difficulty because such manifolds are torsion free.

Proof of THEOREM 4. As before, let $\nu: M \rightarrow X_{r}$ be the normal map induced by the $X$-structure of the $(4 k-1)$-dimensional manifold $M$. Let $p$ be a prime number such that $H_{2 k-1}(X)$ contains no $p$-torsion.

By Theorem IV.2.1 of [1] (see particularly Proposition IV.3.12), we can surger $M$ to a manifold $M_{1}$, so that the map $\nu_{1}: M_{1} \rightarrow X_{r}$ is an isomorphism in homology up to dimension $(2 k-2)$, and the map

$$
\left(\nu_{1}\right)_{*}: H_{2 k-1}\left(M_{1} ; Z_{p}\right) \rightarrow H_{2 k-1}\left(X ; Z_{p}\right)
$$

is a monomorphism. Consider the following commutative diagram

$$
\begin{aligned}
& 0 \rightarrow H_{2 k-1}\left(M_{1}\right) \otimes Z_{p} \rightarrow H_{2 k-1}\left(M_{1} ; Z_{p}\right) \\
& \downarrow \quad \downarrow \\
& 0 \rightarrow H_{2 k-1}(X) \otimes Z_{p} \rightarrow H_{2 k-1}\left(X ; Z_{p}\right)
\end{aligned}
$$

where the horizontal maps are monomorphisms by the universal coefficient theorem, and the second vertical map is a monomorphism as explained before. But this implies that the first vertical map is a monomorphism, and the fact that $H_{2 k-1}(X)$ contains no $p$-torsion implies that the same is true for $H_{2 k-1}\left(M_{1}\right)$. That ends the proof.

I do not know what happens in dimension 3.

I am grateful to the editor for pointing out that the argument on p. 107 of Browder's book [1], does not carry in the $(4 k-1)$-dimensional case.

\section{REFERENCES}

1. W. Browder, Surgery on simply connected manifolds, Springer-Verlag, Berlin and New York, 1972.

2. R. Lashof, Poincaré duality and cobordism, Trans. Amer. Math. Soc. 109 (1963), 257-277.

3. R. Stong, Torsion in manifolds and the semicharacteristic, Indiana Univ. Math. J. 25 (1976), 989-993.

Mathematical Institute, University of Athens, Solonos 57, Athens 143, Greece

Current address: Department of Mathematics, University of Crete, Iraklion, Crete, Greece 